\title{
GROWTH OF GaN METALORGANIC CHEMICAL VAPOUR DEPOSITION LAYERS ON GaN SINGLE CRYSTALS*
}

\author{
K. Pakula, J.M. Baranowski, R. Stępniewski, A. Wysmolek \\ Institute of Experimental Physics, University of Warsaw \\ Hoża 69, 00-681 Warszawa, Poland \\ I. Grzegory, J. Jun, S. Porowski
}

High Pressure Research Center, Polish Academy of Sciences

Sokolowska 29/37, 01-142 Warszawa, Poland

\section{SAWICKI}

Institute of Physics, Polish Academy of Sciences

Al. Lotników 32/46, 02-668 Warszawa, Poland

AND K. STAROWIEYSKI

Chemistry Department, Warsaw Technical University

Koszykowa 75, 00-662 Warszawa, Poland

The homoepitaxial growth of GaN layers has been achieved for the first time. Bulk GaN single crystals which have been used as a substrate have been grown from diluted solution of atomic nitrogen in the liquid gallium at $1600^{\circ} \mathrm{C}$ and at nitrogen pressure of about $15-20 \mathrm{kbar}$. It is shown that a terrace growth of $\mathrm{GaN}$ epitaxial layer has been realized. The high quality of the GaN film has been confirmed by luminescence measurements. The analysis of donor-acceptor and exciton luminescence is presented.

PACS numbers: 78.55.Cr, 78.66.Fd

Recent advances in heteroepitaxy growth of GaN by metalorganic chemical vapour deposition (MOCVD) have demonstrated that high quality films can be grown on sapphire substrates [1]. In this paper we report the homoepitaxial MOCVD growth of GaN using for the first time bulk GaN single crystals as substrates. Bulk $\mathrm{GaN}$ single crystals used as substrates were grown from diluted solution of atomic nitrogen in the liquid gallium at temperature $1600^{\circ} \mathrm{C}$ and at the nitrogen pressure of about $15-20 \mathrm{kbar}$ by the method described previously [2]. The

*This work was supported by the State Committee for Scientific Research (Republic of Poland) grant PBZ-101.12. 
bulk GaN crystals grown by this method had electron concentration well above $10^{19} \mathrm{~cm}^{-3}$, most likely resulting from a high concentration of nitrogen vacancies [3]. In spite of a high concentration of point defects the structural quality of GaN single crystals is very good. The width of X-ray rocking curve for (004) $\mathrm{Cu} K_{\alpha}$ reflection was about 30 arcsec. The presented in this paper results correspond to the GaN layer grown on the GaN platelet of an area of about $5 \mathrm{~mm}^{2}$ with a relatively flat surface. Such single crystal sample has been used for MOCVD growth without any mechanical or chemical treatment. The GaN epitaxial layers have been grown in a horizontal atmospheric pressure MOCVD Epigress system adapted for the nitrides growth. The trimethylgallium (TMG) and $\mathrm{NH}_{3}$ have been used as sources of $\mathrm{Ga}$ and $\mathrm{N}$ respectively, in addition to $\mathrm{H}_{2}$ as carrier gas. The growth took place at temperatures close to $1000^{\circ} \mathrm{C}$ and the flow of gases have been adjusted in such way that a layer of about $2 \mu \mathrm{m}$ thickness was expected. The growth of GaN layer was realized directly on single crystal substrate without deposition of a low temperature nucleation layer.

The scanning electron microscopy micrographs of the surface of the GaN layer are shown in Fig. 1. The terrace growth of GaN was observed and is shown in Fig. 1a. The higher magnification of the GaN single crystal terrace of the thickness of about $1 \mu \mathrm{m}$ is shown in Fig. 1b. On the contrary, GaN layers grown in the same conditions on sapphire substrate have shown hexagonal islands characteristic of the columnar growth.

a)

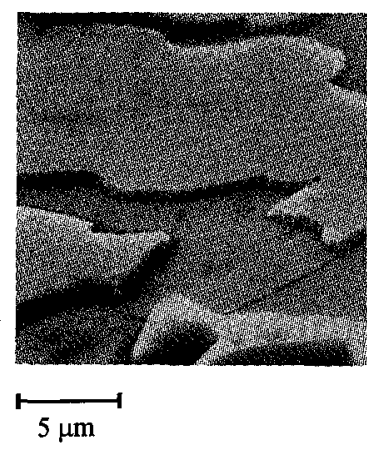

b)

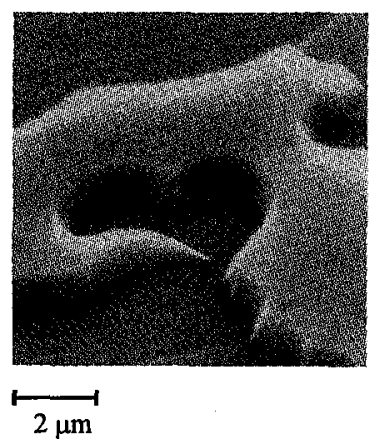

Fig. 1. Scanning electron microscopy micrograph of a low (a) and a high magnification (b) of GaN epitaxial terraces grown on the GaN substrate.

The better quality of GaN layers grown on GaN single crystal is confirmed by the luminescence measurements. The luminescence spectrum of bulk GaN substrate and the spectrum of GaN layer taken at $4.2 \mathrm{~K}$ are qualitatively compared in Fig. 2. Due to high concentration of point defects bulk GaN crystals have a weak and broad band to band luminescence and pronounced yellow luminescence centred at $2.3 \mathrm{eV}$. On the other hand, the GaN layer grown on the GaN substrate shows two orders of magnitude stronger luminescence in the ultraviolet and blue region and practically no luminescence in the yellow region. 


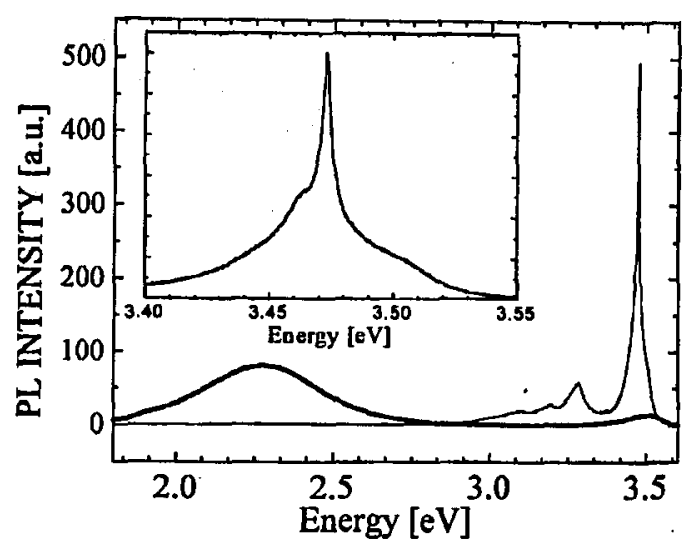

Fig. 2. Luminescence of the GaN single crystal substrate and the GaN epitaxial layer taken at $4.2 \mathrm{~K}$. In the inset the luminescence of the bound exciton region measured on the $\mathrm{GaN}$ epitaxial layer at $4.2 \mathrm{~K}$ is shown.

The blue photoluminescence band consisting of three peaks with the strongest one at $3.28 \mathrm{eV}$ is the same as previously reported [4] and it is most likely due to donor-acceptor pair recombination with an involvement of LO phonon. The temperature dependence of the intensity of the $3.28 \mathrm{eV}$ band gives activation energy of about $100 \pm 10 \mathrm{meV}$. This activation energy may be connected with the ionization energy of a donor active in formation of the donor-acceptor pairs. From the $3.28 \mathrm{eV}$ pair recombination it is possible to estimate the ionization energy of the acceptor as well. The free exciton energy has been recently determined to be equal to $3.485 \mathrm{eV}$ [1]. Using the electron and hole effective masses equal to $m_{\mathrm{e}}^{*}=0.2 m_{0}$ [5] and $m_{\mathrm{h}}^{*}=0.80 m_{0}[6]$ respectively and dielectric constant 5.8 [6], the binding energy of the free exciton of $65 \mathrm{meV}$ and the energy gap of $\mathrm{GaN}$ of about $3.55 \mathrm{eV}$ is obtained. In this case the effective mass hydrogenic donor and acceptor should have the energy of $81 \mathrm{meV}$ and $323 \mathrm{meV}$ respectively. The determined $100 \mathrm{meV}$ ionization energy of the donor indicates the chemical shift of the ground state of about $20 \mathrm{meV}$. However, the ionization energy of the acceptor is only $170 \mathrm{meV}$ which is much smaller than the effective mass hydrogenic acceptor energy. Therefore it seems that the $170 \mathrm{meV}$ acceptor is due to a structural defect and has a localized character.

The most intense luminescence of the GaN layer is observed in the ultraviolet region in a form of a sharp but of an unusual shape line at the energy of $3.473 \mathrm{eV}$ and is shown in the inset to Fig. 2. The line at the $3.472 \mathrm{eV}$ has been previously assigned to the exciton bound to a neutral donor [7]. Taking into account the value of the free exciton energy one gets the binding energy of the bound exciton equal to about $12 \mathrm{meV}$. The $3.473 \mathrm{eV}$ exciton line is seen only at low temperatures in the temperature range between $4.2 \mathrm{~K}$ and $60 \mathrm{~K}$. The temperature dependence of the bound exciton line gives an activation energy of about $10 \mathrm{meV}$, which is in a reasonable agreement with the estimated $12 \mathrm{meV}$ value. 
In addition, this energy is of the order of $10 \%$ of the ionization energy of the donor in a reasonable agreement with Haynes rule [8]. Therefore, it seems that the $3.473 \mathrm{eV}$ line is due to the bound exciton connected with the $100 \mathrm{meV}$ donor. In - addition to the bound exciton line, there is a shoulder on the low energy side of the line and a puzzling peak and tail on the high energy side. The low energy side structure at the energy $3.463 \mathrm{eV}$ may be due to the exciton bound to a neutral acceptor or another neutral donor. The high energy tail with the broad peak at $3.508 \mathrm{eV}$ seems more difficult to explain. The temperature dependence of the high energy shoulder is similar to the $3.473 \mathrm{eV}$ bound exciton line. Therefore the presence of such structure may be connected with existence of excitons related to holes originating from the deeper spin-orbit and crystal field split valence band. The recent reflectivity measurements revealed existence of free excitons at $3.486 \mathrm{eV}, 3.494 \mathrm{eV}$ and $3.520 \mathrm{eV}$ [1]. The energy separation between the lowest and the highest energy exciton is about $34 \mathrm{meV}$. The high energy broad peak at $3.508 \mathrm{eV}$ is also $35 \mathrm{meV}$ above the bound exciton line at $3.473 \mathrm{eV}$, which would suggest that it is due to the bound exciton connected with the split off valence band. The relatively large width of the $3.508 \mathrm{eV}$ peak could originate from the short lifetime of the corresponding exciton and from the background of free electron-hole recombination. The presence of the bound exciton connected with the split valence bands may additionally indicate a good structural quality of the GaN layer. On the other hand, the lack of luminescence connected with free excitons is probably due to relatively large concentration of donors in the GaN layer.

\section{References}

[1] W. Shan, T.J. Schmidt, X.H. Yang, S.J. Hwang, J.J. Song, B. Goldberg, Appl. Phys. Lett. 66, 985 (1995).

[2] S. Porowski, J. Jun, M. Boćkowski, M. Leszczyński, S. Krukowski, M. Wróblewski, B. Eucznik, I. Grzegory, Proc. 8lh Conf. on Semi-Insulating III-V Materials, Warsaw 1994, Ed. M. Godlewski, World Scientific, Singapore 1994, p. 61.

[3] D. Jenkins, J.D. Dow, Ming-hsiung Tsai, J. Appl. Phys. 72, 4130 (1992).

[4] J.A. Freitas, Jr., M.A. Khan, Mater. Res. Soc. Symp. Proc. 339, 547 (1994).

[5] P. Perlin, T. Suski, to be published.

[6] Y. Ueta, S. Sakai, Y. Kamiya, H. Sato, Mater. Res. Soc. Symp. Proc. 339, 459 (1994).

[7] M.A. Khan, D.T. Olson, J.N. Kuznia, W.E. Carlos, J.A. Freitas, Jr., J. Appl. Phys. 74, 5901 (1993).

[8] A.M. Stoneham, Theory of Defects in Solids, Clarendon Press, Oxford 1975, p. 840. 